\title{
The effect of the topology on the spatial ultimatum game
}

\author{
M. N. Kuperman and S. Risau Gusman \\ Centro Atómico Bariloche and Instituto Balseiro, 8400 S. C. de Bariloche, Argentina \\ Consejo Nacional de Investigaciones Cientificas y Técnicas, Argentina
}

\begin{abstract}
In this work we present an analysis of a spatially non homogeneous ultimatum game. By considering different underlying topologies as substrates on top of which the game takes place we obtain nontrivial behaviors for the evolution of the strategies of the players. We analyze separately the effect of the size of the neighborhood and the spatial structure. Whereas this last effect is the most significant one, we show that even for disordered networks and provided the neighborhood of each site is small, the results can be significantly different from those obtained in the case of fully connected networks.
\end{abstract}

\section{INTRODUCTION}

In the last years, Game Theory has been recognized as a powerful alternative way to examining economics [1, 2]. The models analyzed under this scheme consists of sets of agents that play a certain game and a set of the strategies that can be used by the agents. Game theory can be understood as a mathematical tool for analyzing and predicting human behavior in strategic situations. In the last years many physicist have directed their attention towards the analysis of several market games $[3,44,5,6,6,7,8]$

The equilibrium analysis assume that all the players display strategic thinking and optimizing behavior. However, it is widely accepted, and has been experimentally shown, that not every player behaves in a rational way. The realization of this difference led to the creation of Behavioral Economics, a branch of economics closely related to the study of the behavior of economical agents rather than economical quantities [9, 10]. One of the most interesting results obtained in this field is the observation that real individuals do not behave according to the classical assumptions of homo economicus [11], a completely rational individual who seeks to optimize his utilities with the least possible cost.

The gap between economics and Game Theory has been bridged by Evolutionary Game Theory, which takes into account the possibility that the strategies of the agents can change following some evolutionary rule. The Evolutionary Game Theory has succeeded in explaining how cooperation can arise in populations playing noncooperative games, i.e. in games where cooperation is possible but is not favored. Included in the group of non-cooperative games with economical interest we find the Prisoner's Dilemma, the Ultimatum Game, bargaining games, etc.

In the present work we focus on some aspects related to the Ultimatum Game. The essential features of this game can be very easily summarized. Two individuals are told that they have the opportunity to split a given amount of externally provided money. One of the individuals is randomly chosen as the first player and to assume the role of the offerer. He/she has to make a one time offer, consisting in indicating how much of the total amount of money is to be given to each player. The other player, as the respondent, has the opportunity to either accept or reject this offer. If the offer is rejected both get nothing. If the offer is accepted, each one gets the accorded part. Both participants are aware of the rules of the game before making any decision.

The Ultimatum Game is a particular case of bargaining. Game theory predicts that the best strategy is to offer an unequal split favoring the offerer. In [12] it is shown that if $\epsilon$ is the lowest allowed partition and given that a rational responder will prefer a small amount to nothing, the best strategy for the offerer is to give just $\epsilon$ and take the rest. But studies made by behavioral economists have shown that most of the time real individuals tend to reject unequal offers. The first studies are described in [13]. Since then there have been extensive studies on the behaviors of the players under different circumstances and within a wide spectra of cultural environments. Their results do not lead to a unique behavioral profile, and in particular they show clearly that human players usually do not act as the homo economicus [13, 14, 15, 16, 17, 18, 19, 20].

It has also been shown ([21]) that inequity aversion may not be a exclusively human feature: brown capuchin monkeys (Cebus Capella) seem to respond negatively to unequal reward distribution in exchanges with a human experimenter.

In order to explore the ultimatum game beyond the "static" formulation by Rubinstein [12], some authors have analyzed the evolutionary ultimatum game $[22,23$, $24,25,26]$. In these works it is shown that when the agents are placed in an ordered network (and therefore constrained to play with, and imitate, only their neighbors) the evolution takes the system to more equitable strategies than predicted by the rational players hypothesis. One natural question that arises is whether this effect is only due to the spatial distribution of the players or it is also due to the fact that the players are restricted to play and imitate only a very small number of agents.

One of the goals of this work is to stress the important role played by the underlying topology. Notice that ordered networks differ from the fully mixed case not only in that agents are connected to a very small set of other agents, but also on the fact that the clustering is much smaller, i.e. the neighbors of a site are not necessarily connected among themselves. In this work we analyze 
these features separately to understand the effect they have on the evolution of strategies.

We show that some field results can be in correspondence with our findings. For example, in [16] it is shown that the behavior of players cannot be univocally associated neither with the rational nor the altruistic attitudes. On the contrary, experiments across different cultural environments show that it is spread over a wide spectra of possibilities. This result was mentioned but not discussed in previous works. At the same time we establish interesting relationships between the outcome of an evolutionary situation and the underlying social topology.

\section{THE MODEL}

The model consists of a set of $N$ players located on a network, which defines the neighborhood of each player, i.e. the subset of the whole population that is available for interaction. Each player $i$ is assigned a playing strategy that consists of a pair of real numbers $\left(o_{i}, a_{i}\right)$ within the interval $[0,1]$. An interaction consists in taking a pair of linked players, and let them play twice, alternating the roles of offerer and respondent. $o_{i}$ is the offer of player $i$ when acting as offerer, and $a_{i}$ is the smallest amount $i$ accepts when acting as respondent. The total sum allotted in each game is 1 .

The temporal evolution of the game is organized into generations. In each generation, each player interacts with all of its neighbors. The payoff of $i$ when playing with $j, w_{i j}$ is

$$
w_{i j}= \begin{cases}1-o_{i}+o_{j} & \text { if } o_{i} \geq a_{i} \text { and } o_{j} \geq a_{i} \\ 1-o_{i} & \text { if } o_{i} \geq a_{i} \text { and } o_{j}<a_{i} \\ o_{j} & \text { if } o_{i}<a_{i} \text { and } o_{j} \geq a_{i} \\ 0 & \text { if } o_{i}<a_{i} \text { and } o_{j}<a_{i}\end{cases}
$$

After each agent has played with its entire neighborhood we compute the cumulative payoff of each individual and consider that a game generation has concluded. It is at this moment that the evolutionary dynamics takes place. In the next generation all the players are replaced by their offspring. The strategy of a site is updated by choosing one strategy in the neighborhood (including the site to be updated) with probability equal to the ratio between the individual cumulative payoff and the total cumulative payoff of all the sites in the neighborhood.

This warrants a competition process in which only the fittest or more successful strategies survive. In the next generation, a new breed of players occupies the sites of the network, with reset payoffs but with strategies determined by the outcome of the previous generation. The fact that a strategy was successful in a given generation does not guarantee its success in the next one, with a different distribution of strategies.

To avoid the system to get stuck in spurious local minima, we add some noise in the form of small mutations, associated to a mutation rate $\epsilon$ [23]. Once the process of reproduction is finished by determining which player will leave its offspring in which sites, the descendants copy their ancestor strategy with a small variation: if the individual that formerly occupied the site $m$ leaves a descendant in the place $l$, the strategy of the new occupant of the site $l$ is then

$$
\left(o_{l}(t+1), a_{l}(t+1)\right)=\left(o_{m}(t)+\delta_{o}, a_{m}(t)+\delta_{a}\right)
$$

with $\delta \in[-\epsilon, \epsilon]$ a real random number.

We have performed simulations in three different topologies: ordered, disordered, and $k$-Small World Networks ( $k$-SWN), to interpolate smoothly between the ordered and disordered topologies. The ordered topology consists of nodes on a ring, joined to their first $k$ neighbors to each side. These networks are highly clustered: many neighbors of each node are connected among themselves, forming triangles. This characteristic is quantified by the clustering coefficient which is the number of triangles centered on each node divided by the number of pairs of neighbors, averaged over all the nodes. The disordered topology we consider is a random graph where all the nodes have the same degree $2 k$ (also called regular random graphs). The third topology is a variation of the small world networks of Watts and Strogatz (WS) 27].

The algorithm of construction of WS networks is as follows: starting from an ordered network, the ring is traversed and with probability $p^{\prime}$ each link is rewired to a random node. Double and self links are not allowed. Though the algorithm conserves the total number of links, at the end of the process the degree of each node is statistically characterized by a binomial distribution, for $p^{\prime}>1$. As we are interested in filtering any effect related to changes in the size of the neighborhoods we modify the original WS algorithm to constrain the resulting networks to a subfamily with a delta shaped degree distribution. We call this family of networks the $k$-Small World Networks ( $k$-SWN), where $2 k$ indicates the degree of the nodes. We start again from an ordered network and define a disorder parameter $p$ that plays a role analogous to that of $p^{\prime}$ in WS networks. To proceed with the reconnection of the network we choose two couples of linked nodes (or partners) rather than one. With probability $p$ we decide whether to switch or not the partners in order to get two new pairs of coupled links. In this way all the nodes preserve their degree while the process of reconnection assures the introduction of a certain degree of disorder. It must be stressed that the dependence with $p$ of the clustering coefficient and path length is qualitatively similar to what is observed as a function of $p^{\prime}$ in SWNs.

\section{NUMERICAL RESULTS}

The simulations were done on networks with $10^{3}-10^{4}$ individuals and different values of $k$. Defining a generation as the situation when each player plays twice (as offerer and as respondent) with all his/her neighbors we 


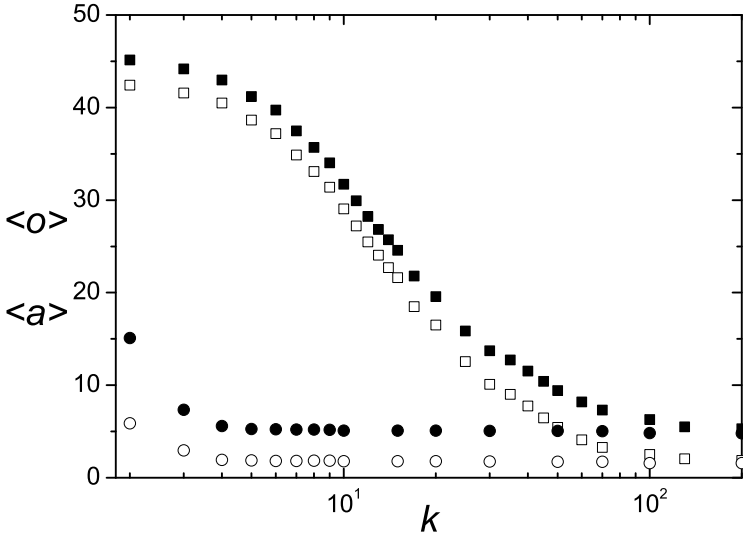

FIG. 1: Asymptotic mean thresholds $\langle o\rangle$ (full symbols) and $\langle a\rangle$ (empty symbols) for agents in a k-small world network, as a function of the node degree $k$. Squares: Ordered Network, Circles: Regular Random Network

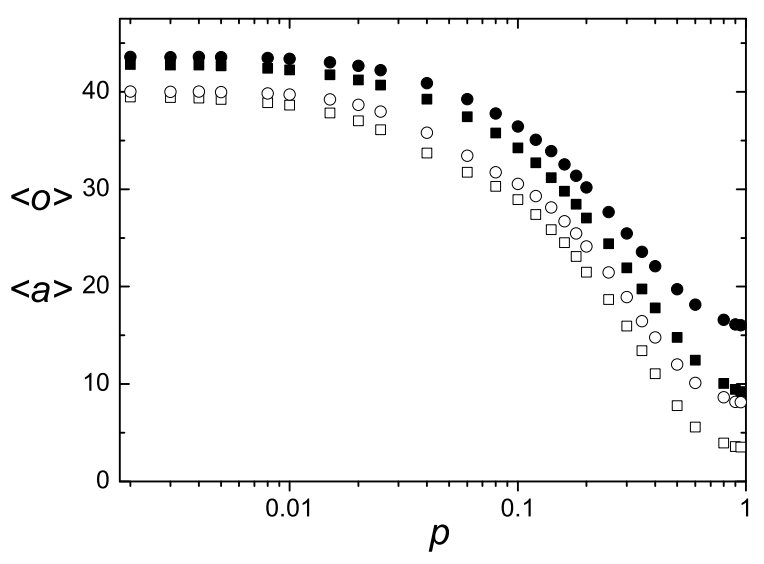

FIG. 2: Asymptotic mean thresholds $\langle o\rangle$ (full symbols) and $\langle a\rangle$ (empty symbols) for agents in a k-small world network, as a function of the disorder parameter of the network. Squares: $k=2$, Circles: $k=3$

performed time averages over the last $10^{6}$ generations of each realization, after a transient equally long. At the same time, the time averaged results for a given set of parameters $p$ and $k$ were obtained after averaging over 100 individual cases.

We begin by showing the comparison (see Fig 1) between the strategies attained by agents placed in a ring, each one connected to its first $k$ neighbors, and the ones attained when playing in a regular random graph (i.e. a graph where each node is connected to exactly $k$ random neighbors). These last networks have a vanishing clustering coefficient, which means that, locally, they are isomorphic to trees. On the other hand, the networks defined on the ring have a clustering that increases with $k: c=\frac{3}{4} \frac{k-2}{k-1}$. It is interesting to see that evolution in a completely disordered network can also lead to strategies that are rather far from the rational prediction, provided that the number of neighbors is small enough. On the other hand, a small increase in the number of neighbors leads to strategies much closer to the rational prediction. This effect is much less pronounced in the case of the ring networks but it must be borne in mind that clustering is also increasing in this case. The effect of clustering can be grasped by comparing the curves for fixed values of $k$ : larger clustering seems to lead to the evolution of more equitable strategies. To analyze this in more detail we have performed simulations of the Ultimatum Game in k-Small World Networks.

As we vary the disorder parameter $p$ we observe a monotonous smooth behavior of the values of $\langle o\rangle$ and $\langle a\rangle$. In Fig. 2 we show the mean values of $\langle o\rangle$ as a function of the disorder parameter $p(\langle a\rangle$ is not shown, as its behavior is very similar). The behavior of this quantity looks qualitatively very similar to that of the clustering coefficient. To stress this fact we have plotted $\langle o\rangle$ vs. $C$ in Fig. 3, where an almost linear dependence can be observed.

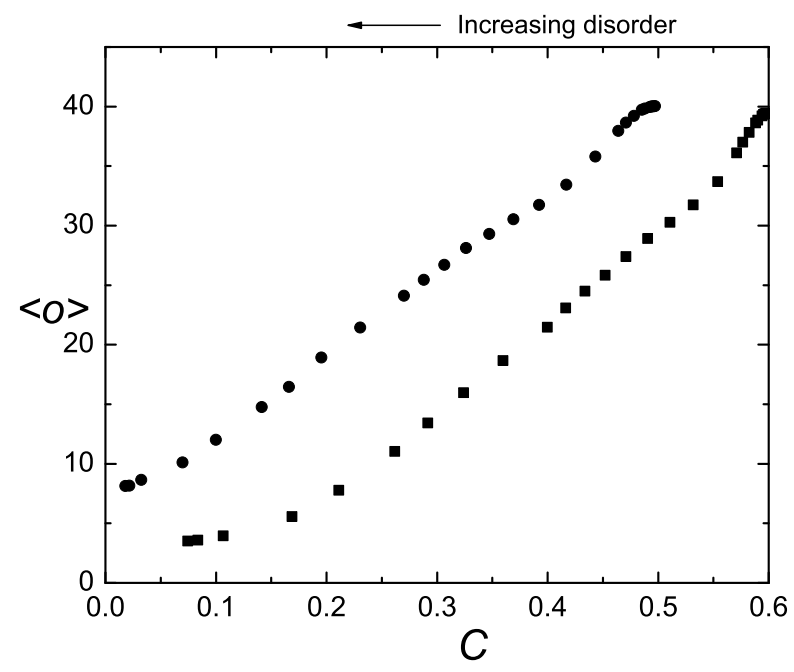

FIG. 3: Asymptotic mean thresholds $\langle o\rangle$ for agents in a k-small world network, as a function of the clustering of the network. Squares: $k=2$, Circles: $k=3$

\section{INVASION ANALYSIS}

When studying the dynamics of systems embedded in ordered or semiordered networks it is difficult to go beyond numerical simulations. Direct analytical approaches usually become impossibly complicated in the 
presence of short loops. For this reasons people have used indirect approaches with the hope of capturing the essence of the problem.

One possibility to study the asymptotic state is to calculate the strategy that a cluster of mutants should have to invade an homogeneous population. The behavior of this as the topology is changed has been shown to give some clues about the asymptotic state of different systems [29, 30]. We have performed these calculations for the limit cases of a completely ordered and a completely disordered network. The calculation is analogous to the one in [22], where only the conditions for invasion of clusters of two and three sites were considered, for the ordered network with $k=2$. In an infinite population of individuals with offer and acceptance thresholds set to $o_{b}$ and $a_{b}$, a cluster of $n$ mutants is introduced, with offer and acceptance thresholds set to $o_{m}$ and $a_{m}$ with $o_{m}>a_{m}>o_{b}>a_{b}$. For this initial state we derive the condition $o<o_{c}(k)$ that must be satisfied for the mutants to leave more than $n$ descendants in the next generation (this is only a first approximation, because mutant expansion might not continue indefinitely [30]). Notice that for a homogeneous population, with mutation allowed, the average thresholds should be bigger that $o_{c}$. Otherwise a mutation could generate a mutant cluster that would invade the population, thus raising the average threshold.

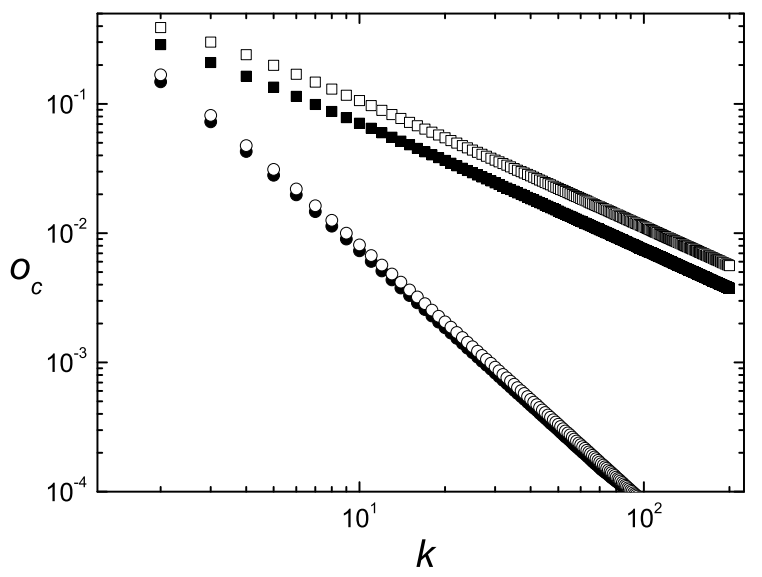

FIG. 4: Critical invasion thresholds as a function of the degree of the nodes and for a cluster size 2 (full symbols) and 3 (empty symbols). Squares: Ordered Network, Circles: Regular Random Network

The behavior of $o_{c}(k)$ for clusters of two and three sites, in ordered and completely disordered networks is shown in Fig 4 . The decrease of both curves with $k$ was to be expected, because if a cluster has a strategy well suited for invading the population, increasing the number of its neighbors can only add to its success. A more interesting feature is the relationship between both curves for the same value of $k$. In this case the clusters have the same number of connections, but they differ in how these connections are arranged: in the completely disordered network all these connections go to different agents, whereas in the ordered network the two members of it are connected mostly to the same sites (i.e. there are more triangles). Thus, the figure shows that acting cooperatively leads to better reproductive chances, and this is favored in networks with larger clustering coefficient. Notice that the average payoff of the agents is the same in the two cases. It is their relative fitness what changes.

The same features are present when one compares the invasion thresholds for larger compact clusters, if their size does not depend on $k$.

\section{CONCLUSIONS}

The rationale of the present work was to reveal the influence of the underlying topology on the outcome of an evolutionary Ultimatum Game. Though previous works have already addressed this aspect, some interesting details could only be unveiled through a more detailed analysis. This task was achieved by making numerical simulations on several types of networks, with different amount of disorder and degree distribution. We have observed that both the increase of the neighborhood size and the increase of the degree of disorder have a similar effect, leading a population of players towards responses with increasing levels of "rationality". As the increment of the size of the neighborhood makes a given network converge towards a fully connected graph and thus being associated to a mean field situation, the effect of the increase on the disorder was not clear up to now. We have shown the transition in the behavior of the population when the underlying topology varies continuously between the extremes already studied in the literature.

The behavior of the asymptotic state of the system as the clustering of the underlying network changes can be compared to that of some games where the evolution of cooperation is analyzed. Individuals with fair strategies can survive provided that they are surrounded by neighbors with similar behavior. A highly clustered network favors this necessary condition and the system naturally evolves to a situation when the mean level of offers is around $50 \%$. On the contrary, when the underlying network present a low level of clusterization, the mean values go to values close to zero. In this case, the transient shows first a diminishing acceptance threshold, that finally drives the offer value to lower values as well, as shown in Figure 5. If we identify the fair strategies as being more cooperative that those with lower offers the results can be interpreted within the frame of the phenomenology of other cooperative games where the spatial distribution plays the same role as here and leads to the same qualitative results.

So far, we have made a description and analysis of the obtained results. After analyzing our results we are in- 


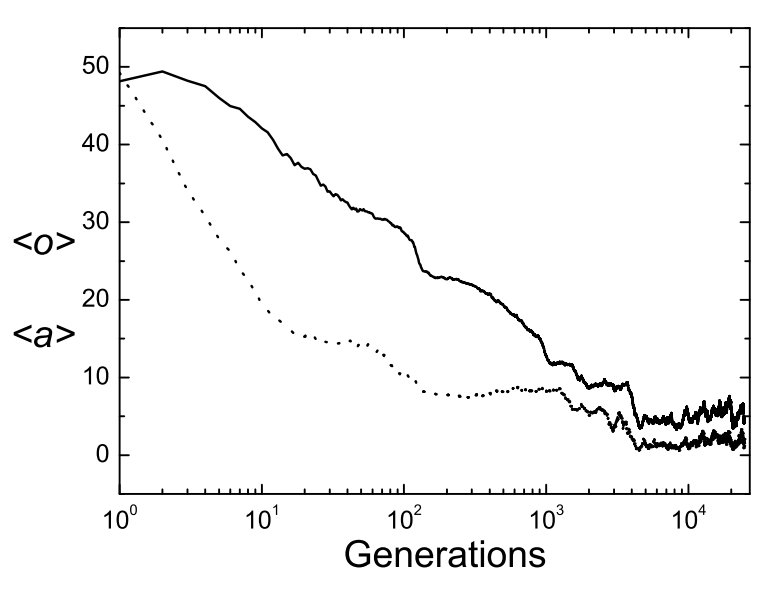

FIG. 5: Temporal behavior of the thresholds $\langle o\rangle$ (full) and $<a\rangle$ (dotted) in the fully connected network

clined to propose an explanation for the emergence of a collective behavior that sets a discrepancy between the theoretical predictions and field results in relation to the ultimatum game. Furthermore, the experimental results show a wide spectra of behaviors that could not be easily explained or unified within a suitable frame. In this work we study an evolutionary process that changes the strategies of the populations and can lead to the survival of the fittest behaviors. The final composition of the evolutionary strategies of the population seems to depend on the social underlying architecture. This could explain, in principle, the fact that people with different social organization display different collective behaviors. The model succeeds to qualitatively reproduce the complexity of the observed experimental results based on very simple assumptions and interaction rules. Summarizing we show that the whole spectra of observed behaviors can be reproduced by considering an evolutionary process that together with the subtleties of the social organization shapes the behavior of different people.

\section{APPENDIX}

We consider a population of agents with offer and acceptance thresholds set to $p_{0}$ and $q_{0}<p_{0}$, with two con- nected mutants with thresholds $p_{m}>q_{m}>p_{0}>q_{0}$. Agents can be connected to none, one, or both mutants, depending on the topology of the network. Their respective average payoff after a round of games will be:

$$
\begin{aligned}
& f_{0}(k)=2 k \\
& f_{1}(k)=2 k-1+p \\
& f_{2}(k)=2 k-2+2 p
\end{aligned}
$$

where $2 k$ is the number of neighbors. For the mutants, the payoff is:

$$
f_{m}(k)=1+(2 k-1)(1-p)
$$

At the next generation, the new strategy for each site is chosen randomly among its own and its neighbors strategies, with a probability proportional to their respective average payoffs. Thus, the expected number of mutants at the next generation is:

$$
\begin{aligned}
m(k)= & \frac{4 f_{m}(k)}{2 f_{m}(k)+(2 k-1) f_{1}(k)}+ \\
& \frac{(2 k-1) f_{m}(k)}{f_{m}(k)+f_{1}(k)+(2 k-1) f_{0}(k)}
\end{aligned}
$$

for the disordered case and

$$
\begin{aligned}
m(k)= & 2 \frac{2 f_{m}(k)}{2 f_{m}(k)+2(k-1) f_{2}(k)+f_{1}(k)}+ \\
& \sum_{j=1}^{k-1} \frac{2 f_{m}(k)}{2 f_{m}(k)+(2 k-2-j) f_{2}(k)+f_{1}(k)+j f_{0}(k)}+ \\
& \frac{f_{m}(k)}{f_{m}(k)+(k-1) f_{2}(k)+f_{1}(k)+k f_{0}(k)}
\end{aligned}
$$

for the completely ordered case. The invasion condition for $p$ can then be extracted from the equation $m(k)>2$.
[1] C. F. Camerer, Behavioral Game Theory (Princeton University Press - 2003).

[2] K. Chatterjee and W.F. Samuelson, Game Theory and Bussiness Aplications(Kuwer Academic Publishing - 2002).

[3] J. Berg and M. Weigt, Europhys. Lett. 48, 129 (1999).

[4] C.L. Tang, W.X. Wang, X. Wu, B.H. Wang, Eur. Phys.
J. B 53, 411 (2006).

[5] Z.G. Huang, Z.X. Wu, X.J. Xu, J.Y. Guan, Y.H. Wang, Eur. Phys. J. B 58, 493 (2007).

[6] J.-Y. Guan, Z.X. Wu, Z.G. Huang, X.J. Xu and Y.H. Wang, Europhys. Lett. 76, 1214 (2006).

[7] M. Perc Europhys. Lett. 75, 841 (2006).

[8] P. Jefferies, M.L. Hart, P.M. Hui and N.F. Johnson, Eur. 
Phys. J. B 20, 493 (2001).

[9] P. Diamond and H Vartiainen (Eds.), Behavioral Economics and Its Applications, (Princeton University Press $-2007)$

[10] C. F. Camerer, G. Loewenstein and M. Rabin (Eds.), Advances in Behavioral Economics (Princeton University Press - 2003).

[11] J. Persky, Journal of Economic Perspectives 9, 221-231 (1995).

[12] A. Rubinstein, Econometrica 50, 97 (1982).

[13] W. Güth, R. Schmittberger and B. Schwarze , An Experimental Analysis of Ultimatum Bargaining, Journal of Economic Behavior \& Organization 3, 367 (1982).

[14] D. Kahneman, J. L. Knetsch, and R. H. Thaler, Fairness and the Assumptions of Economics, Journal of Business 59, S285 (1986).

[15] D. Kahneman, J. L. Knetsch, and R. H. Thaler, Fairness as a Constraint on Profit Seeking: Entitlements in the Market, American Economic Reuiew 76, 728 (1986).

[16] J. Henrich, R. Boyd, S. Bowles, C. Camerer, E. Fehr, H. Gintis and R. McElreath, In search of homo economicus: Behavioral experiments in 15 small-scale societies, AER Papers and Proceedings 91, 73-78 (2001).

[17] L.R. Anderson, Y. V. Rodgers, and R.R. Rodriguez, Cultural differences in attitudes towards bargaining, Economics Letters 69, 45 (2000).

[18] J. Henrich, Does culture matter in economic behavior?
Ultimatum game bargaining among the Machiguenga of the Peruvian Amazon, American Economic Review 90, 973 (2000).

[19] S. J. Solnick, Gender differences in the ultimatum game, Economic Inquiry 39, 189 (2001).

[20] T. Brenner and N. J. Vriend, On the behavior of proposers in ultimatum games, Journal of Economic Behavior \& Organization 61617 (2006)

[21] S.F. Brosnan and F. B. M. de Waal, Nature 425, 297 (2003).

[22] K. M. Page, M. A. Nowak and K. Sigmund, Proc. R. Soc. Lond. B 267, 2177 (2000)

[23] M. A. Nowak, K. Page and K. Sigmund. Science 289, 1773 (2000).

[24] K. Page and M. A. Nowak. J. theor. Biol. 209, 173 (2000).

[25] T. Killingback and E. Studer, Proc. Biol. Sci. 268, 1797 (2001).

[26] A. Sánchez and J. A. Cuesta, J. Theor. Biol. 235, 233(2005).

[27] D. J. Watts and S. H. Strogatz, Nature 393, 440 (1998).

[28] S. Milgram, Psychol. Today 2, 60 (1967).

[29] T. Killingback, M. Doebeli and Nancy Knowlton, Proc. R. Soc. Lond. B 266, 1723 (1999).

[30] Ch. Hauert, Proc. R. Soc. Lond. B 268, 761 (2001). 